\title{
Fractal algorithm for the modeling of consumption in COVID-19
}

\section{Algoritmo Fractal para la modelación del consumo en COVID-19}

\author{
RAMOS-ESCAMILLA, María†* \\ ECORFAN-Mexico, S.C. \\ ID $1^{\text {st }}$ Author: María, Ramos-Escamilla / ORC ID: 0000-0003-0865-8846, Researcher ID Thomson: J-7654-2017, CVU \\ CONACYT ID: 349660
}

DOI: 10.35429/JMQM.2020.6.4.1.7

Received March 28, 2020; Accepted June 30, 2020

\begin{abstract}
We present an analysis of the exogenous factors of consumption, we simulate it in a fractal algorithm whose objective is the Brownian equilibrium through the iterative multiplication of assumptions of an initial function at current prices whose values are constant and positive that will derive in the final solution that is characterized by non-negativity and does not denote absolute convergence, only relative to their economies of scale compared to iterative methods, we find other procedures derived from the stochastic method but formulated strictly as mathematical developments, they are fractal optimization algorithms, which are based on search of an objective function that minimizes the distance between the initial function and the expected iterations of the candidate functions to be a solution, verifying the corresponding restrictions.
\end{abstract}

Fractal, COVID-19, Consumption

\begin{abstract}
Resumen
Presentamos un análisis de los factores exógenos del consumo lo simulamos en un algoritmo fractal cuyo objetivo es el equilibrado browniano mediante la multiplicación iterativa de supuestos de una función inicial a precios corrientes cuyos valores son constantes y positivos que derivarán en la solución final que se caracteriza por la no negatividad y no denota convergencia absoluta, solo relativa respecto de sus economías a escala frente a los métodos iterativos encontramos otros procedimientos derivados del método estocástico pero formulados estrictamente como desarrollos matemáticos, se trata de algoritmos de optimización fractal, que se basan en la búsqueda de una función objetivo que minimice la distancia entre la función inicial y las iteraciones esperadas de las funciones candidatas a ser solución, verificando las restricciones correspondientes.
\end{abstract}

Fractal, COVID-19, Consumo

Citation: RAMOS-ESCAMILLA, María. Fractal algorithm for the modeling of consumption in COVID-19. Journal-Mathematical and Quantitative Methods. 2020. 4-6: 1-7

\footnotetext{
* Correspondence to Author (email: ramos@ecorfan.org)

$\uparrow$ Researcher contributing first author
} 


\section{Introduction}

The use of calibration in the static modeling of the traditional economy originates from the need to use complex models with economic policy objectives that simulate the affected short and long-term shock. For this, it is necessary to use as a starting point models that are theoretically consistent but that are intensive in the use of parameters.

The first step for the construction of the model is to have consistent micro information for a certain period and it will be assumed that the economy is in equilibrium. Then, it is necessary to perform a calibration of the model, in this procedure the values of the parameters are estimated. Once all of them have been obtained and the model specified, the model is replicated, checking that an equilibrium is reached with the estimated parameters. Now, it is possible to evaluate the effects of different policies by comparing the reference equilibrium with the simulated one.

Calibration can be understood as the process by which parameter values are inferred from economic data of a given period, and that once those values are specified in an applied model, the data of the base period are endogenously replicated as a solution of the same. To represent the economy, the information must be structured within a scheme that ensures compliance with certain sectoral consistency $^{1}$ requirements $\varphi_{1} \rightarrow \varphi_{n} \quad$ and macroeconomic $(N)$.

$\varphi_{1}(x)+\varphi_{2}(x)+\cdots+\varphi_{N}(x)=0\left(\frac{3}{2}(\log N) N^{\frac{1}{2}}\right)+\varepsilon$

\footnotetext{
${ }^{1}$ To add two complex numbers, add the real parts and add the imaginary parts: $(\mathrm{V}+\mathrm{i} \cdot \mathrm{w})+(\mathrm{x}+\mathrm{i} \cdot \mathrm{y})=(\mathrm{v}+\mathrm{x})+\mathrm{i}$. $(w+y)$. The product of two complex numbers $(v+i \cdot w)$ - $(\mathrm{x}+\mathrm{i} \cdot \mathrm{y})$ can be obtained by multiplying binomials, remembering i $2=-1$. Grouping the roots and the imaginary parts of the products, we obtain: $(\mathrm{V}+\mathrm{i} \cdot \mathrm{w}) \cdot(\mathrm{x}$ $+\mathrm{i} \cdot \mathrm{y})=(\mathrm{v} \cdot \mathrm{x}-\mathrm{w} \cdot \mathrm{y})+\mathrm{i} \cdot(\mathrm{v}+\mathrm{w} \cdot \mathrm{y} \cdot \mathrm{x})$, here we limit the relationship between the iteration formula of $\mathrm{z}$ and those of $X$ and $Y$, now it should be clear. These complex numbers are usually very simple in nature and originate in successive iterations sets of a certain dimension, fixed throughout the process that is modified when the iteration becomes infinite.

${ }^{2}$ Gauss Criterion: Let be a series of positive terms $\sum a_{n}$, is calculated:
}

$$
\lim _{n \rightarrow \infty} \frac{a_{n}}{a_{n-1}}=L
$$

\section{Consumer microeconomics}

We will begin a process that comprises five phases:

Formulation of the problem (quantifiable) to investigate $(\mathrm{P}, \mathrm{t})$, current prices, this conditions the type of model that could be used and therefore the information requirements necessary to find the answer between the available products $\mathrm{t}>3.8$ and those used $\lim _{t \rightarrow 0}$.

$\frac{\partial}{\partial t} y(P, t)=C[y(P, t)], t>3.8$

$\lim _{t \rightarrow 0} y(P, t)=y_{0}(P)$

$\frac{d}{d t} y(t)=C[y(t)], t>3.8$

$\lim _{t \rightarrow 0}|| y(t)-y_{0}||=3.8$ Selection of a type of theory (already tested). Theories may be adequate for some facts and not for others. In particular, the general equilibrium theory of the consumer with respect to production can be modified to adapt it to different closings.

Neoclassical closure ${ }^{2}$ :

$\frac{d}{d t}\left[C(t) p_{0}\right]=C\left[T(t) p_{0}\right], t>3.8$

$\lim _{t \rightarrow 0}|| C(t) p_{0}-p \|=3.8$

Keynesian closure $^{3}$ :

||$C\left(t ; p_{0}\right)|| \leq C e^{t}|| p_{0}||, t>3.8$

Structuralist closure ${ }^{4}$ :

If $\mathrm{L}<1$ the series is convergent, if $\mathrm{L}>1$ the series is divergent and, if $\mathrm{L}=1$ the criterion does not allow to determine convergence or divergence.

${ }^{3}$ Cauchy Criterion: Let be a series of positive terms $\sum a_{n}$ , is calculated: $\lim _{n \rightarrow \infty} \sqrt[n]{a_{n}}=L$

If $\mathrm{L}<1$ the series is convergent, if $\mathrm{L}>1$ the series is divergent and, if $\mathrm{L}=1$ the criterion does not allow to determine convergence or divergence.

${ }^{4}$ Gamma Criterion: Let be a series of positive terms $\sum a_{n}$ and $\sum \frac{1}{u_{n}}$ a divergent series. Is calculated:

$$
\lim _{n \rightarrow \infty}\left(u_{n} \frac{a_{n}}{a_{n+1}}-u_{n+1}\right)=L
$$

If $\mathrm{L}>0$ the series $\sum a_{n}$ converges, if $\mathrm{L}<0$ the series $\sum a_{n}$ diverges and the comparison series is deformed geometric series (fractal evidence). 


$$
C\left\{\int_{S} x(p) d p=\int_{S} C[x(p)] d p\right.
$$

Choice of functional shapes and model resolution ${ }^{5}$. What is important is not whether the model is realistic or not, but whether it is capable of providing a quantitative answer to a specific question that the researcher asks.

$\sum_{i} C_{k} x\left(p_{k}\right), \sum_{i} C_{k} C\left[x\left(P_{k}\right)\right]=C\left\{\sum_{i} p_{k} x\left(P_{k}\right)\right.$

Gray's code applies to (a, b, and c) because it is based on a permutation of traditional binary code, it provides a representation of ordered objects such that, going from one object to the next, we only have to change one bit of information. The Hamming distance between the representation of one object and the next (or the predecessor) is 1 .

We start by performing smoothed functionality for each market period 2020 (X), $2021(\mathrm{~J})$ :

$(\theta n+1, X n+1)=f(\theta n+, X n)=(\theta n+$ $\alpha \sin 6 \theta n-b \sin 4 \theta n-X n \sin \theta n-J \cos \theta n)$

According to the price run, we obtain:

For the Ex Ante (2020): $\mathrm{q}_{\mathrm{n}+1}=\mathrm{f}\left(\mathrm{p}_{\mathrm{n}}\right)+\mathrm{u}_{\mathrm{n}}$

For the Ex Post (2021): $p_{n+1}=q_{n+1}+r_{n}$

Level of competition: $\mathrm{p}_{\mathrm{H}} \mathrm{V}_{1}^{\mu}\left(\mathrm{p}_{1}-\mathrm{V}_{2}^{\mathrm{v}}\left(\mathrm{p}_{2}\right)-\right.$ $\mathrm{H}\left(\mathrm{p}_{\mathrm{H}}\right)$

We demonstrate the market in competition:

$$
\begin{aligned}
& \Gamma_{\mu v}^{\mathrm{HV}_{1} \mathrm{~V}_{2}}\left(\mathrm{PH}, \mathrm{P}_{1}, \mathrm{P}_{2}\right)=\delta \mathrm{zM}_{\mathrm{Z}}^{2}\left[\mathrm{~h}_{1}^{\mathrm{V}_{1} \mathrm{~V}_{2}} \delta_{\mu \mathrm{v}}+\frac{\mathrm{h}_{2}^{\mathrm{V}_{1} \mathrm{~V}_{2}}}{\mathrm{M}_{\mathrm{z}}^{2}} \mathrm{P}_{2 \mu} \mathrm{P}_{1 \mathrm{v}}\right] \\
& \mathrm{h} \frac{2}{\pi}\left(\mathrm{P}_{1} \cdot \mathrm{P}_{2}\right)=\frac{\mathrm{P}_{1}^{2}+\mathrm{P}_{2}^{2}-\mathrm{m}_{\mathrm{H}}^{2}}{\mathrm{~m}_{\mathrm{Z}}^{2}} \mathrm{C}_{2} \mathrm{Z}_{\mathrm{y}}-\frac{\mathrm{P}_{1}^{2}-\mathrm{P}_{2}^{2}-\mathrm{m}_{\mathrm{M}}^{2}}{\mathrm{~m}_{\mathrm{Z}}^{2}} \mathrm{C}_{3} \mathrm{Z}_{\mathrm{y}}
\end{aligned}
$$

Combination of productive factors:

$\left(\mathrm{e}+\mathrm{e}^{-} \rightarrow \tau \bar{\tau} \mathrm{y}\right)=$

$\int \frac{\alpha^{3}}{\mathrm{e}}\left[\mathrm{m}_{\tau}^{2} \mathrm{C}_{1}\left(\mathrm{x}_{\mathrm{w}}\right)\left[\mathrm{F}_{1}\left(\mathrm{~s}, \mathrm{E}_{\gamma}, \cos \theta_{\gamma}\right)\left(\mathrm{h}_{2}^{\mathrm{Z}_{\gamma}}\right)^{2}\right]\right]$

\footnotetext{
${ }^{5}$ If the terms of a series of positive terms are less than or equal to those of another convergent series, it is convergent ${ }^{5}$. Let be $\sum a_{k}$ a string whose character you want to set and is $\sum_{k=1}^{\infty} u_{k}$ a convergent series, with sum $\mathrm{U}$, verifying that $a_{k} \leq \mathrm{u}_{\mathrm{k}}$, so $\sum a_{k}$ converges and its sum S is less than or equal to the sum $U$. The Serie $\sum_{k=1}^{\infty} u_{k}$ is a larger series than the given series.
}

$$
\begin{aligned}
& +\mathrm{m}_{\mathrm{r}}^{2} \mathrm{C}_{2}\left(\mathrm{x}_{\mathrm{w}}\right)\left[\mathrm{F}_{3}\left(\mathrm{~s}, \mathrm{E}_{\gamma}, \cos \theta_{\gamma}\right) \mathrm{h}_{1}^{\mathrm{Z \gamma}}+\right. \\
& \left.\mathrm{F}_{4}\left(\mathrm{~s}, \mathrm{E}_{\gamma}, \cos \theta_{\gamma}\right) \mathrm{h}_{2}^{\mathrm{Z}_{\gamma}}\right] \\
& \left.+\mathrm{C}_{3}\left(\mathrm{x}_{\mathrm{w}}\right) \mathrm{F}_{5}\left(\mathrm{~s}, \mathrm{E}_{\gamma}, \cos \theta_{\gamma}\right)\right] \mathrm{E}_{\gamma} \mathrm{dE_{ \gamma }} \mathrm{d} \cos \theta_{\gamma}
\end{aligned}
$$

Exogenous risks to productivity and competitiveness in Mexico:

$$
\begin{aligned}
& \mathrm{F}_{1}\left(\mathrm{~s}, \mathrm{E}_{\mathrm{y}}, \cos \theta_{\mathrm{y}}\right) \equiv \\
& \frac{1}{2} \frac{\left(\frac{1}{2} \mathrm{~s}-\sqrt{\mathrm{s}} \mathrm{E}_{\mathrm{y}}-2 \mathrm{~m}_{\mathrm{Z}}^{2}\right)}{\left[\left(\mathrm{s}-\mathrm{M}_{\mathrm{Z}}^{2}\right)^{2}+\mathrm{M}_{\mathrm{Z}}^{2} \Gamma_{\mathrm{Z}}^{2}\right]\left(\mathrm{s}+2 \sqrt{\mathrm{s}} \mathrm{E}_{\mathrm{y}}-\mathrm{M}_{\overline{\mathrm{H}}}^{2}\right)^{2}} \\
& \mathrm{~F}_{2}\left(\mathrm{~s}, \mathrm{E}_{\mathrm{y}}, \cos \theta_{\mathrm{y}}\right) \equiv \frac{1}{3}\left[\left(\mathrm{~s}-\mathrm{M}_{\mathrm{Z}}^{2}\right)^{2}+\mathrm{M}_{\mathrm{Z}}^{2} \Gamma \frac{2}{\mathrm{z}}\right](\mathrm{s}+ \\
& \left.2 \sqrt{\mathrm{s}} \mathrm{E}_{\mathrm{y}}-\mathrm{M} \frac{2}{\mathrm{H}}\right)^{2} \\
& \mathrm{~F}_{3}\left(\mathrm{~s}, \mathrm{E}_{\mathrm{y}}, \cos \theta_{\mathrm{y}}\right) \equiv \frac{1}{5}\left[\left(\mathrm{~s}-\mathrm{M}_{\mathrm{Z}}^{2}\right)^{2}+\mathrm{M}_{\mathrm{Z}}^{2} \Gamma \frac{2}{\mathrm{Z}}\right](\mathrm{s}+ \\
& \left.2 \sqrt{\mathrm{s}} \mathrm{E}_{\mathrm{y}}-\mathrm{M} \frac{2}{\mathrm{H}}\right) \\
& \mathrm{F}_{4}\left(\mathrm{~s}, \mathrm{E}_{\mathrm{y}}, \cos \theta_{\mathrm{y}}\right) \equiv \frac{1}{8}\left[\left(\mathrm{~s}-\mathrm{M}_{\mathrm{Z}}^{2}\right)^{2}+\mathrm{M}_{\mathrm{Z}}^{2} \Gamma \frac{2}{\mathrm{z}}\right](\mathrm{s}+ \\
& \left.2 \sqrt{\mathrm{s}} \mathrm{E}_{\mathrm{y}}-\mathrm{M} \frac{2}{\mathrm{H}}\right) \\
& \mathrm{F}_{5}\left(\mathrm{~s}, \mathrm{E}_{\mathrm{y}}, \cos \theta_{\mathrm{y}}\right) \equiv \frac{1}{11} \frac{\left[\left(4-\sin ^{2} \theta_{\mathrm{y}}\right) \sqrt{\mathrm{s}} \sin ^{2} \theta_{\mathrm{y}}\right.}{\left[\left(\mathrm{s}-\mathrm{M}_{\mathrm{Z}}^{2}\right)^{2}+\mathrm{M}_{\mathrm{Z}}^{2} \Gamma_{\mathrm{Z}}^{2}\right]\left(\sqrt{\mathrm{s}} \sin ^{2} \theta_{\mathrm{y}}\right.}
\end{aligned}
$$

We integrate the masses and determine the investment levels to determine growth:

Low level:

$$
C_{1}\left(x_{W}\right) \equiv \frac{\left(1-5 x_{W}+10 x_{W}^{2}\right)}{x_{W}^{3}\left(1-x_{W}\right)^{3}}
$$

Medium level:

$$
C_{2}\left(x_{w}\right) \equiv \frac{\left(1-5 x_{w}\left(1-5 x_{w}+10 x_{w}^{2}\right)\right.}{x_{w}^{5 / 2}\left(1-x_{w}\right)^{5 / 2}}
$$

High level:

$$
C_{3}\left(x_{W}\right) \equiv \frac{\left(1-5 x_{w}+10 x_{w}^{2}\right)^{2}}{x_{w}^{8 / 2}\left(1-x_{w}\right)^{8 / 2}}
$$

Similarly, it can be said that, if the terms of a series of positive terms are greater than or equal to those of another divergent series, it is divergent. 
We determine the supports: i) Ex Ante Price: $F$ and ii) Ex Post Price: G

$$
\{\mathrm{F}, \mathrm{G}\}\{\mathrm{z}\}=\frac{\partial \mathrm{F}}{\partial_{\mathrm{qi}}} \frac{\partial \mathrm{G}}{\partial_{\mathrm{pi}}}(\mathrm{z})-\frac{\partial \mathrm{G}}{\partial_{\mathrm{qi}}} \frac{\partial \mathrm{F}}{\partial_{\mathrm{pi}}}(\mathrm{z})=1
$$

\section{Impact of COVID-19 on international consumption}

In today's global economy, trade barriers are as follows:

- $\quad$ Tariff barriers and excessive customs procedures;

- $\quad$ Restrictions on access to raw materials;

The obstacles to the exchange of services and foreign direct investment;

Restrictive practices regarding public contracts;

The use of unfair or discriminatory tax practices (State aid, subsidies and methods incompatible with WTO rules for trade defense, such as anti-dumping measures);

The incorrect use of unjustified measures in terms of health, safety and technical regulations;

Insufficient protection and nonapplication of intellectual property rights (IPR). These obstacles to trade are characterized by their complexity and difficulty in detecting them.

Thus, non-tariff barriers and other "internal" barriers are becoming increasingly important. Many market access problems that have arisen are explained by the fact that the existing rules are not properly applied. Considering the Hénon-Heiles system for expected IFS [Frame, M., Johnson, B., Sauerberg, J: 2000]:

We limit the scaling levels [Frame, M., Philip, A., G., D, Robucci, A: 1992] of the productive markets:

$\{\mathrm{F}, \mathrm{G}\}=\frac{\partial \mathrm{F}}{\partial_{\mathrm{qi}}} \frac{\partial \mathrm{G}}{\partial_{\mathrm{pi}}}-\frac{\partial \mathrm{F}}{\partial_{\mathrm{pi}}} \frac{\partial \mathrm{G}}{\partial_{\mathrm{qi}}}=\frac{\partial \mathrm{F}}{\partial \mathrm{q}_{\mathrm{i}}} \frac{\partial \mathrm{qi}_{\mathrm{i}}}{\partial \mathrm{F}}+\frac{\partial \mathrm{F}}{\partial_{\mathrm{qi}}} \frac{\partial \mathrm{pi}}{\partial \mathrm{F}}=\frac{\mathrm{dF}}{\mathrm{dF}}=1$
$\{\mathrm{F}, \mathrm{G}\}=\frac{\partial \mathrm{F}}{\partial_{\mathrm{qi}}} \frac{\partial \mathrm{G}}{\partial_{\mathrm{pi}}}-\frac{\partial \mathrm{F}}{\partial_{\mathrm{pi}}} \frac{\partial \mathrm{G}}{\partial_{\mathrm{qi}}}=\frac{\partial \mathrm{p}_{\mathrm{i}}}{\partial \mathrm{G}} \frac{\partial \mathrm{G}}{\partial \mathrm{p}_{\mathrm{i}}}+\frac{\partial \mathrm{qi}}{\partial \mathrm{G}} \frac{\partial \mathrm{G}}{\partial \mathrm{q}_{\mathrm{i}}}=\frac{\mathrm{dG}}{\mathrm{dG}}=1$

We multiply the vectors:

$\frac{\partial \mathrm{p}_{\mathrm{i}}}{\partial_{\mathrm{G}}}=\frac{\partial \mathrm{F}}{\partial_{\mathrm{qi}}}, \frac{\partial \mathrm{p}_{\mathrm{i}}}{\partial_{\mathrm{G}}}=\frac{\partial \mathrm{F}}{\partial_{\mathrm{p}_{\mathrm{i}}}}$

Orthogonality of geospatial vectors [Barnsley, M., J. S. Geronimo, A., N. Harrington: 1982]:

$\mathrm{X}_{\mathrm{F}}=\left(-\frac{\partial \mathrm{F}}{\partial_{\mathrm{p}}}, \frac{\partial \mathrm{F}}{\partial_{\mathrm{q}}}\right), \mathrm{X}_{\mathrm{G}}\left(-\frac{\partial \mathrm{G}}{\partial_{\mathrm{p}}}, \frac{\partial \mathrm{G}}{\partial_{\mathrm{q}}}\right)$

$\mathrm{X}_{\mathrm{F}} \cdot \mathrm{X}_{\mathrm{G}}=-\frac{\partial \mathrm{F}}{\partial_{\mathrm{p}_{\mathrm{i}}}} \frac{\partial \mathrm{G}}{\partial_{\mathrm{p}_{\mathrm{i}}}}-\frac{\partial \mathrm{F}}{\partial_{\mathrm{q}_{\mathrm{i}}}} \frac{\partial \mathrm{G}}{\partial_{\mathrm{q}_{\mathrm{i}}}}$

$\nabla \mathrm{X}_{\mathrm{F}}=-\frac{\partial}{\partial_{\mathrm{q}_{\mathrm{i}}}} \frac{\partial \mathrm{F}}{\partial_{\mathrm{p}_{\mathrm{i}}}}+\frac{\partial}{\partial_{\mathrm{p}_{\mathrm{i}}}} \frac{\partial \mathrm{F}}{\partial_{\mathrm{q}_{\mathrm{i}}}}=0$

$\nabla \mathrm{X}_{\mathrm{G}}=-\frac{\partial}{\partial_{\mathrm{q}_{\mathrm{i}}}} \frac{\partial \mathrm{G}}{\partial_{\mathrm{p}_{\mathrm{i}}}}-\frac{\partial}{\partial_{\mathrm{p}_{\mathrm{i}}}} \frac{\partial \mathrm{G}}{\partial_{\mathrm{q}_{\mathrm{i}}}}=0$

Field of attraction of shares on inverse products:

$\frac{\mathrm{dF}}{\mathrm{dG}}=\frac{\partial \mathrm{F}}{\partial_{\mathrm{q}_{\mathrm{i}}}} \frac{\partial \mathrm{q}_{\mathrm{i}}}{\partial \mathrm{G}}+\frac{\partial \mathrm{F}}{\partial_{\mathrm{p}_{\mathrm{i}}}} \frac{\partial \mathrm{p}_{\mathrm{i}}}{\partial \mathrm{G}}=\frac{\partial \mathrm{p}_{\mathrm{i}}}{\partial \mathrm{G}} \frac{\partial \mathrm{q}_{\mathrm{i}}}{\partial \mathrm{G}}-\frac{\partial \mathrm{q}_{\mathrm{i}}}{\partial \mathrm{G}} \frac{\partial \mathrm{p}_{\mathrm{i}}}{\partial \mathrm{G}}=0$

$\frac{\mathrm{dG}}{\mathrm{dF}}=\frac{\partial \mathrm{G}}{\partial \mathrm{q}_{\mathrm{i}}} \frac{\partial \mathrm{q}_{\mathrm{i}}}{\partial \mathrm{F}}+\frac{\partial \mathrm{G}}{\partial \mathrm{p}_{\mathrm{i}}} \frac{\partial \mathrm{p}_{\mathrm{i}}}{\partial \mathrm{F}}=\frac{\partial \mathrm{p}_{\mathrm{i}}}{\partial \mathrm{F}} \frac{\partial \mathrm{q}_{\mathrm{i}}}{\partial \mathrm{F}}-\frac{\partial \mathrm{q}_{\mathrm{i}}}{\partial \mathrm{F}} \frac{\partial \mathrm{p}_{\mathrm{i}}}{\partial \mathrm{F}}=0$

Changes in the prices of production:

$\{$ F. G $\}-\left(\frac{\partial \mathrm{G}}{\partial_{\mathrm{P}_{\mathrm{i}}}} \frac{\partial}{\partial_{\mathrm{q}_{1}}}-\frac{\partial \mathrm{G}}{\partial_{\mathrm{q}_{\mathrm{i}}}} \frac{\partial}{\partial_{\mathrm{P}_{\mathrm{i}}}} \mid\right) \mathrm{F}-\mathscr{L}_{\mathrm{G}^{\mathrm{F}}}-\left[\mathscr{L}_{\mathrm{G}^{\mathrm{F}}}\right]-1$

$\{\mathrm{F} . \mathrm{G}\}-\left(\frac{\partial \mathrm{F}}{\partial_{\mathrm{q}_{\mathrm{i}}}} \frac{\partial}{\partial_{\mathrm{p}_{1}}}-\frac{\partial \mathrm{F}}{\partial_{\mathrm{p}_{\mathrm{i}}}} \frac{\partial}{\partial_{\mathrm{q}_{\mathrm{i}}}} \mid\right) \mathrm{G}-\mathscr{L}_{\mathrm{F}^{\mathrm{G}}}=\left[\mathscr{L}_{\mathrm{F}, \mathrm{G}}\right]=1$

Cognition function:

$\mathscr{L}_{\mathrm{F}}=\frac{\partial \mathrm{F}}{\partial_{\mathrm{p}_{\mathrm{i}}}} \frac{\partial}{\partial_{\mathrm{q}_{1}}}+\frac{\partial \mathrm{F}}{\partial_{\mathrm{q}_{\mathrm{i}}}} \frac{\partial}{\partial_{\mathrm{p}_{\mathrm{i}}}}=\mathrm{X}_{\mathrm{F}} \cdot \nabla$

Participation function:

$\mathscr{L}_{\mathrm{G}}=\frac{\partial \mathrm{G}}{\partial_{\mathrm{p}_{\mathrm{i}}}} \frac{\partial}{\partial_{\mathrm{q}_{1}}}-\frac{\partial \mathrm{G}}{\partial_{\mathrm{q}_{\mathrm{i}}}} \frac{\partial}{\partial_{\mathrm{p}_{\mathrm{i}}}} \equiv \mathrm{X}_{\mathrm{G}} \cdot \nabla$

Reverse derivations:

$\left[\mathscr{L}_{\mathrm{F}}, \mathrm{u}(\mathrm{z})\right]=\mathscr{L}_{\mathrm{F}}, \mathrm{u}(\mathrm{z})=\mathrm{x}_{\mathrm{F}} \cdot \nabla \mathrm{u}(\mathrm{z})=\frac{\mathrm{d} \mathrm{z}}{\mathrm{dg}} \cdot \nabla \mathrm{u}(\mathrm{z})=\frac{\mathrm{du}(\mathrm{z})}{\mathrm{dg}}$

$\left[\mathscr{L}_{\mathrm{G}}, \mathrm{u}(\mathrm{z})\right]=\mathscr{L}_{\mathrm{G}}, \mathrm{u}(\mathrm{z})=\mathrm{x}_{\mathrm{G}} \cdot \nabla \mathrm{u}(\mathrm{z})=\frac{\mathrm{dz}}{\mathrm{df}} \cdot \nabla \mathrm{u}(\mathrm{z})=\frac{\mathrm{du}(\mathrm{z})}{\mathrm{df}}$

RAMOS-ESCAMILLA, María. Fractal algorithm for the modeling of consumption in COVID-19. JournalMathematical and Quantitative Methods. 2020 


$$
\begin{aligned}
& \mathrm{u}(\mathrm{z} ; \mathrm{g})=\mathrm{e}^{8^{\mathscr{F}} \mathrm{F}} \mathrm{u}(\mathrm{z}), \mathrm{u}(\mathrm{z} ; \mathrm{f})=\mathrm{e}^{\mathrm{f}^{\mathscr{G} \mathrm{G}}} \mathrm{u}(\mathrm{z}) \\
& {\left[\mathscr{L} \frac{\mathrm{m}}{\mathrm{G}}, \mathrm{F}\right]=\mathrm{m} \mathscr{\mathscr { m } - 1} \frac{\mathrm{G}}{\mathrm{G}}, \quad\left[\mathscr{L}_{\mathrm{G}}, \mathrm{F}^{\mathrm{n}}\right]=\mathrm{nF}^{\mathrm{n}-1}} \\
& {\left[\mathscr{L} \frac{\mathrm{m}}{\mathrm{F}}, \mathrm{G}\right]=\mathrm{m} \mathscr{\mathscr { m } - 1} \frac{\mathrm{F}}{\mathrm{F}}, \quad\left[\mathscr{L}_{\mathrm{F}}, \mathrm{G}^{\mathrm{n}}\right]=\mathrm{nG}^{\mathrm{n}-1}} \\
& \mathrm{~L}_{1,2,3}=\left(\begin{array}{c}
\mathrm{e} \\
\mathrm{v}_{\mathrm{e}} \\
\mathrm{e}^{\mathrm{c}}
\end{array}\right)_{\mathrm{L}},\left(\begin{array}{c}
\mu \\
\mathrm{v}_{\mu} \\
\mu^{\mathrm{c}}
\end{array}\right)_{\mathrm{L}},\left(\begin{array}{c}
\tau \\
\mathrm{v}_{\tau} \\
\tau^{\mathrm{c}}
\end{array}\right)_{\mathrm{L}}
\end{aligned}
$$

Super-price scales:

$$
\emptyset_{\gamma}=\left(\begin{array}{l}
\emptyset_{\gamma} \\
\emptyset^{0}
\end{array}\right): \emptyset_{1}=\left(\begin{array}{l}
\emptyset_{1} \\
\delta^{-}
\end{array}\right): \emptyset_{2}=\left(\begin{array}{c}
\emptyset_{2} \\
\mathrm{P}^{--}
\end{array}\right)
$$

Spectral results:

Future-2021:

$$
\mathrm{Y}_{\mu}^{++}=\frac{1}{\sqrt{2}}\left(\mathrm{~A}_{\mu}^{1}-\mathrm{i} \mathrm{A}_{\mu}^{2}\right)
$$

Present-2020:

$$
\mathrm{Y}_{\mu}^{+}=\frac{1}{\sqrt{2}}\left(\mathrm{~A}_{\mu}^{3}-\mathrm{i} A_{\mu}^{5}\right)
$$

\section{Past-2019:}

$$
\mathrm{W}_{\mu-}=\frac{1}{\sqrt{2}}\left(\mathrm{~A}_{\mu}^{8}-\mathrm{i} \mathrm{A}_{\mu}^{11}\right)
$$

Scaling amplitudes:

$\mathcal{M}_{7 T T V^{0}}=\sum_{\mathrm{x}} \mathcal{M}_{77 T V^{0}}^{\mu_{1} \mu_{2} \mu_{3} \mu_{4}} \epsilon_{\mu_{1}}\left(\mathrm{p}_{1}, \lambda_{1}\right) \epsilon_{\mu_{2}}\left(\mathrm{p}_{2}, \lambda_{2}\right) \epsilon_{\mu_{3}}\left(\mathrm{p}_{3}, \lambda_{3}\right) \epsilon_{\mu_{4}}\left(\mathrm{p}_{4}, \lambda_{4}\right)$

Fractal self-similarity [Mandelbrot, B. B., Vespignani, A., Kaufman, H: 1995]:

$$
\mathcal{M}_{\frac{1}{2}}=\sum_{\mathrm{F}} \mathcal{M}_{\mathrm{F}} \mathcal{M}_{1}=\sum_{\mathrm{V}} \mathcal{M}_{\mathrm{v}} \mathcal{M}_{0}=\sum_{\mathrm{S}, \mathrm{H}} \mathcal{M}_{\mathrm{S}, \mathrm{H}}
$$

Fractal self-affinity [Mandelbrot, B. B., Vespignani, A., Kaufman, H: 1995]:

$$
\begin{aligned}
& \mathrm{P} 1_{\mu 1}=\sum_{\mathrm{X}} \mathcal{M}_{777 \mathrm{~V}^{0}}^{\mu_{1} \mu_{2} \mu_{3} \mu_{4}}=0 \\
& \mathrm{P} 2_{\mu 2}=\sum_{\mathrm{X}} \mathcal{M}_{777 \mathrm{~V}^{0}}^{\mu_{1} \mu_{2} \mu_{3} \mu_{4}}=0
\end{aligned}
$$

\footnotetext{
${ }^{6}$ Synthetic economies:

$\int_{x}^{\infty} p(n) d n\left[\left(\frac{1}{2} x\right) \frac{x}{\sqrt{2 \sigma}}\right]$

$p_{e}^{B}=\frac{1}{2}\left(\sqrt{\frac{3 E_{b}}{N_{0}}}\right)+\frac{1}{\sqrt{12 \pi \frac{E_{b}}{N_{0}}}}(1-E)^{-3 E_{b}} / N_{0}$
}

The limit of $1 / 2$ at constant $d x$ :

$$
\mathrm{P} 3_{\mu 3}=\sum_{\mathrm{X}} \mathcal{M}_{777 \mathrm{~V}^{0}}^{\mu_{1} \mu_{2} \mu_{3} \mu_{4}}=0
$$

\section{Aggregate consumption for Mexico}

The parameterization and reproduction of known theoretical results. In general, a question has a theoretically known answer, and the model should give an approximately correct answer to this question. Choose a series of questions to check in the next stage.

Likewise, the data is used to calibrate $\left[p(t) p_{0}\right]-C^{\prime}(t) p_{0} d p$ a model economy ${ }^{6}$ in such a way as to reproduce the real economy as much as possible.

$\int_{\alpha}^{\beta} e^{-\lambda t} C\left[p(t) p_{0}\right] d p=\int_{\alpha}^{\beta} e^{-\lambda t} C^{\prime}(t) p_{0} d p$

$$
\begin{aligned}
& =e^{-\lambda \beta} C\left(\beta ; p_{0}\right)-e^{-\lambda \beta} C\left(\alpha ; p_{0}\right)+ \\
& \lambda \int_{\alpha}^{\beta} e^{-\lambda t}\left[c t ; p_{0}\right] d(c, p)
\end{aligned}
$$

Once the functional forms of the production and preference functions have been decided, having assigned the values to the parameters and, in the case of stochastic models [Frame, M., Neger, N: 2010], using the probability distribution for shocks, iterations can be carried out.

$C \int_{\alpha}^{\beta} e^{-\lambda t} c\left[p t ; p_{0}\right] d t=\int_{\alpha}^{\beta} e^{-\lambda t} C y\left[t ; p_{0}\right] d t$

Calibrated models have long been used in other disciplines resulting in an interactive game between theoretical developments and attempts to evaluate whether the new Ex Ante theoretical structures $t_{R}$, A priori $b_{R}$, Ex Post $\mathrm{J}_{3 \mathrm{R}}$ :

Ex Ante:

$$
\rho=\left(\begin{array}{c}
\mathrm{G}_{\mathrm{W}}^{+} \\
\frac{\mathrm{iG}_{\mathrm{z}}+\mathrm{V}}{\sqrt{2}} \\
0
\end{array}\right)_{\mathrm{L}}
$$

$p_{e}^{M}=\int_{0}^{1 / 2} \frac{1}{\pi \sqrt{(1-x)}}[(1-2 x)] \sqrt{2 \frac{E_{b}}{N_{0}}} d x$

$\mathrm{p}_{\mathrm{e}}^{f}=\int_{0}^{\frac{1}{2}} \frac{1}{\sqrt{\left(x+\frac{1}{2}\right)\left(\frac{1}{2}-x\right)}}$

RAMOS-ESCAMILLA, María. Fractal algorithm for the modeling of consumption in COVID-19. JournalMathematical and Quantitative Methods. 2020 
A priori:

$\eta=\left(\begin{array}{c}\frac{\mathrm{iG}_{\mathrm{z}}+\mathrm{V}}{\sqrt{2}} \\ -\mathrm{G}_{\mathrm{W}}^{-} \\ 0\end{array}\right)$

Ex Post:

$\mathrm{x}=\left(\begin{array}{c}\mathrm{G}_{\mathrm{Y}}^{-} \\ \mathrm{G}_{\mathrm{x}}^{--} \\ \frac{\mathrm{w}+\mathrm{iG}_{\mathrm{z}^{\prime}}}{\sqrt{2}}\end{array}\right)$

\section{Conclusions}

For these estimates to make sense $0 \leq \kappa<1$. In the real model, values of $\kappa$ greater than one would be equivalent to matrices whose inclination is greater than 45 degrees and the time between consecutive impacts associated with the system follows a geometric law of ratio $0<$ in $<1$ and therefore, there is an accumulation of impacts in finite time that we will call stop time and enter the dynamics of $T^{\frac{1}{2}}$ :

$\mathrm{L}=\int \mathrm{d}^{4} \mathrm{xdy}\left(\mathcal{L}_{\mathrm{F}}+\mathcal{L}_{\mathrm{Y}}\right)$

Sticking with Fourier:

$\mathrm{B}_{\mu}(\mathrm{x}, \mathrm{y})=\frac{1}{\sqrt{\pi \mathrm{R}}} \mathrm{B}_{\mu}^{(0)}(\mathrm{x})+\frac{\sqrt{2}}{\sqrt{\pi \mathrm{R}}}$

On the sidelines:

$\mathrm{Q}(\mathrm{x}, \mathrm{y})=\frac{1}{\sqrt{\pi \mathrm{R}}} \mathrm{Q}_{\mathrm{L}}^{(0)}(\mathrm{x})+$

$\frac{\sqrt{2}}{\sqrt{\pi \mathrm{R}}} \sum_{\mathrm{n}=1}^{\infty} \mathrm{B}_{\mu}^{(\mathrm{n})}(\mathrm{x}) \cos \left(\frac{\mathrm{n} \pi \mathrm{y}}{\mathrm{R}}\right) \mathrm{B}(\mathrm{x}, \mathrm{y})=$

$\frac{\sqrt{2}}{\sqrt{\pi \mathrm{R}}} \sum_{\mathrm{n}=1}^{\infty} \mathrm{B}_{5}^{(\mathrm{n})}(\mathrm{x}) \sin \left(\frac{\mathrm{n} \pi \mathrm{y}}{\mathrm{R}}\right)$

At cost:

$\mathrm{Q}(\mathrm{x}, \mathrm{y})=\frac{1}{\sqrt{\pi \mathrm{R}}} \mathrm{Q}_{\mathrm{L}}^{(0)}(\mathrm{x})+$

$\frac{\sqrt{2}}{\sqrt{\pi \mathrm{R}}} \sum_{\mathrm{n}=1}^{\infty}\left[\mathrm{Q}_{\mathrm{L}}^{(\mathrm{n})}(\mathrm{x}) \cos \left(\frac{\mathrm{n} \pi \mathrm{y}}{\mathrm{R}}\right)+\mathrm{Q}_{\mathrm{R}}^{(\mathrm{n})} \sin \left(\frac{\mathrm{n} \pi \mathrm{y}}{\mathrm{R}}\right)\right]$

$\mathrm{U}(\mathrm{x}, \mathrm{y})=\frac{1}{\sqrt{\pi \mathrm{R}}} \mathrm{U}_{\mathrm{R}}^{(0)}(\mathrm{x})+$

$\frac{\sqrt{2}}{\sqrt{\pi \mathrm{R}}} \sum_{\mathrm{n}=1}^{\infty}\left[\mathrm{Q}_{\mathrm{tR}}^{(\mathrm{n})}(\mathrm{x}) \cos \left(\frac{\mathrm{n} \pi \mathrm{y}}{\mathrm{R}}\right)+\mathrm{U}_{\mathrm{tL}}^{(\mathrm{n})} \sin \left(\frac{\mathrm{n} \pi \mathrm{y}}{\mathrm{R}}\right)\right]$
They serve to represent the real observations through the application of the best assignments of the values of the parameters to the theoretical structures Ex Ante and Ex Post of consumption considering the COVID-19 risk, if we take into account the interrelationships between all the variables considered, which allows capturing its direct and indirect effects, thus overcoming the Brownian equilibrium approaches [Barnsley, M: 1984], which consider only the relevant market of the sector analyzed for Mexico.

$$
\begin{aligned}
& E\left(v_{2}, v_{2}\right)-E\left(v_{2}, v_{1}\right)-E\left(v_{1}, v_{2}\right)- \\
& E\left(v_{1}, v_{1}\right) \geq 3.8
\end{aligned}
$$

Internal consistency among all variables, taking into account macroeconomic balances, sectoral balances of supply and demand and institutional balances of sources and uses of funds to maximize activity in the external sector (F) in terms of exports, therefore we limit the Ex Ante and Ex Post partitions.

\section{References}

Mandelbrot B.( 1972). Lognormal hypothesis and distribution of energy dissipation in intermittent turbulence.Statistical Models \& Turbulence (La Jolla, CA, 1972), 333-351

Mandelbrot B. (1974). Intermittent turbulence in self-similar cascades: divergence of high moments and dimension of the carrier. Journal of Fluid Mechanics, 62, 331-358

Mandelbrot B. (1974). Iterated random multiplications and invariance under randomly weighted averaging. Translated from Comptes Rendus (Paris) 278A, 289-292 \& 355-358

Mandelbrot B. (1975) Limit theorems on the self-normalized bridge range. $\mathrm{Z}$. Wahrscheinlichkeitstheorie verw. Gebiete: 31, 271-285.

Mandelbrot B. (1975). Geometry of homogeneous scalar turbulence: iso-surface fractal dimensions 5/2 and 8/3. J. Fluid Mech, Part 2,72, 401-416

Mandelbrot B.( 1975). Earth's relief, shape and fractal dimension of coastlines, and number-area rule for islands. Proc. National Academy of Sciences (USA) 72, 3825-3838 
Mandelbrot B. y Kampé J. (1965).calcul des probabilités et climatologiqe statistique.Une classe de processus stochastiques homothétiques á soi; application á la loi climatologique de H. E. Hurst.C.R.Acad. Sc, 260, 3274-3277

Mandelbrot B., Pierre J.y Lévy P. (Ed.). (1965).Analyse mathématique. Ensembles de multiplicité aléatoires. C.R.Acad. Sc, 261, 39313933

Mandelbrot B.y Mandelbrojt S. (Ed.). (1974).Calcul des probabilités.Multiplications aléatoires itérées et distributions invariantes par moyenne pondérée aléatoire C.R.Acad. Sc., 278.

Mandelbrot B. y Mandelbrojt S (Ed.). (1974). Calcul des probabilités.Multiplications aléatoires itérées et distributions invariantes par moyenne pondérée aléatoire: quelques extensions C.R.Acad. Sc., 278.

Mandelbrot B.y Mandelbrojt S. (Ed.). (1975).Calcul des probabilités.Fonctions aléatoires pluri-temporelles; approimation poissonienne du cas brownien et généralisations. C.R.Acad. Sc., 280.

Mandelbrot B. Lich. (Ed.).(1975).Statistique céleste.Sur un modéle décomposable d' Univers hiérarchisé: deduction des corrélations galactiques sur la sphére céleste. C.R.Acad. Sc., 280.

Mandelbrot B.y Leray J.(1976) Mécanique des fluids.Géométrie fractale de la turbulence.Dimension de Hausdorff, dispersion et nature des singularités du mouvement des fluides C.R.Acad. Sc., 282.

Mandelbrot B.y Mandelbrojt S. (1978) Calcul des probabilités et statistique des polyméres.Colliers aléatoires et une alternative aux promedades au hasard sans boucle: les cordonnets discrets et fractals. C.R.Acad. Sc., 286.

Mandelbrot B. y Mandelbrojt S. (1979) Statistique céleste.Correlations et texture dans un nouveau modéle d' Univers hiérarchisé, base sur leas ensembles trémas. C.R. Acad. Sc., 288. 